\title{
Uso del aprendizaje cooperativo en educación física y su relación con la responsabilidad individual en estudiantes del nivel secundario Use of cooperative learning in physical education and its relationship with individual responsibility in high school students
}

\author{
*Rudy Gilberto López Guillén, **Josué Taveras Sandoval \\ *Instituto Superior de Formación Docente Salomé Ureña (República Dominicana), **Escuela Ana Josefa Jiménez Yépez \\ (República Dominicana)
}

Resumen. El presente artículo tiene como objetivo comparar el uso del aprendizaje cooperativo en la clase de educación física del nivel secundario de dos centros educativos y verificar su relación con la responsabilidad individual. El estudio fue cuasiexperimental, solo con post test. Después de confirmar las características psicométricas del Cuestionario de Aprendizaje Cooperativo, se hicieron comparaciones de los puntajes obtenidos en las escalas del cuestionario entre el grupo experimental y el grupo control. La hipótesis planteada fue que los participantes del grupo experimental obtendrían puntuaciones más altas que los del grupo control en el factor Responsabilidad Individual. Los participantes del grupo experimental fueron 104 estudiantes, quienes participaron en una unidad didáctica cooperativa y luego llenaron el cuestionario. Los participantes del grupo control fueron 154 estudiantes, quienes no participaron de una unidad didáctica cooperativa, pero sí llenaron el cuestionario. Para poder validar las escalas del cuestionario se realizó un Análisis Factorial Confirmatorio (AFC) con el software Amos 22 para comprobar que se mantenían los 5 factores propuestos originalmente. Encontrando como resultado que las medias de puntuaciones en el cuestionario siempre fueran más altas en el grupo experimental. Como era de esperar, la única diferencia estadísticamente significativa fue encontrada en el factor Responsabilidad Individual. Lo cual lleva a la conclusión de que, las clases cooperativas sí mejoraron notablemente una competencia necesaria para un mejor aprendizaje. Palabras claves. Aprendizaje cooperativo, educación física, responsabilidad individual, competencias, enseñanza secundaria.

\begin{abstract}
The objective of this article is to compare the use of cooperative learning in the physical education class at the high school level of two educational centers and to verify its relationship with individual responsibility. The study was quasiexperimental, with post-test only. In addition to confirming the psychometric characteristics of the Cooperative Learning Questionnaire we compared the scores obtained on the scales of the questionnaire between the experimental group and the control group. The expected hypothesis was that the participants in the experimental group would obtain higher scores than those in the control group on the Individual Responsibility factor. The participants of the experimental group were 104 students, who participated in a cooperative teaching unit and then filled out the questionnaire. The control group participants were 154 students, who did not participate in a cooperative teaching unit but did fill out the questionnaire. To validate the questionnaire scales, a Confirmatory Factor Analysis (CFA) was performed with the Amos 22 software to verify that the 5 originally proposed factors were maintained. As a result, we found that the mean scores in the questionnaire were always higher in the experimental group. As expected, the only statistically significant difference was found in the Individual Responsibility factor. This leads to the conclusion that cooperative classes did significantly improve one necessary skill for better learning.
\end{abstract}

Key words. Cooperative learning, physical education, individual responsibility, competences, secondary school.

\section{Introducción}

El aprendizaje cooperativo (AC) es una metodología mediante la cual los estudiantes se sienten más cautivados por las numerosas características que a él se asocian. Velázquez-Callado en el (2015) define el AC, como «un modelo pedagógico en el que los estudiantes trabajan juntos en pequeños grupos, generalmente

Fecha recepción: 08-08-20. Fecha de aceptación: 06-06-21

Rudy Gilberto López Guillén

rudygil12@gmail.com heterogéneos, para maximizar su propio aprendizaje y también el del resto de compañeros y compañeras» (p.25). Además de la motivación que crea en los niños el trabajo en equipo, alcanzar una meta común es lo que les debe mover, trabajar sin segregar ni desplazar a nadie, ya que el objetivo principal de la cooperación es alcanzar un mismo fin en el cual todos hayan sido partícipes. Pero el simple hecho de ubicar estudiantes en grupos y decirles que trabajen juntos no da como resultado un AC. La diferencia del modelo pedagógico mencionado frente al trabajo grupal es esencialmente la responsabilidad compartida en el aprendizaje. El estudian- 
te asume un papel activo, facilitándole la ayuda necesaria al resto de sus compañeros para que logren la meta en común y no se limita solamente a aprender un contenido. (Arís, 2015; García, Sánchez \& Ferriz, 2020, Martínez-Benito, 2017; Navarro, Pellicer \& Collado 2020; Pérez \& Hortigüela, 2019).

Siguiendo el mismo orden de ideas, otros autores nos dicen que en el $\mathrm{AC}$ el éxito de un grupo depende del éxito mutuo entre los miembros del grupo, con la especificidad de que estos deben ser heterogéneos y los estudiantes no deben perder el aprendizaje individual (Anderman \& Dawson, 2011). De hecho, en el aula ideal todos los estudiantes aprenderían cómo trabajar en cooperación con otros, sin dejar de trabajar autónomamente. Esto significa que los estudiantes siempre trabajan motivados, pues la única forma en que los miembros del grupo pueden alcanzar sus propios objetivos personales es si el grupo tiene éxito. Por lo tanto, para alcanzar sus metas personales los miembros del grupo deben ayudar a sus compañeros a hacer lo que sea necesario para lograr que el grupo tenga éxito y, quizás aún más importante, alentar a sus compañeros a esforzarse al máximo (Bermejo et al., 2020).

Es preciso señalar lo que nos comenta Pujolàs (2012), cuando dice que «el cambio en la estructura de la actividad individualista o competitiva por otra de tipo cooperativo contribuye a que los estudiantes puedan aprender juntos, en un aula inclusiva, sean cuales sean sus necesidades educativas y sus culturas de origen» (p.111). Con esta metodología los estudiantes no compiten por el simple objetivo de ganar, sino que compiten para jugar, amenizar y alegrarse (Feria-Madueño, GrimaldiPuyana, Sánchez-Oliver, \& Alcaraz-Rodríguez, 2017; Johnson, Johnson, \& Smith, 2014).

\section{El AC en educación física}

La educación física tiene unos modelos de enseñanza básicos y otros emergentes, los cuales son: «aprendizaje cooperativo, educación deportiva, comprensivo de iniciación deportiva, responsabilidad personal y social, educación aventura, alfabetización motora, estilo actitudinal, modelo ludo técnico, autoconstrucción de materiales y educación para la salud» (Fernández-Río, Calderón, Hortigüela, Pérez-Pueyo, \& Aznar, 2016, p. 55). Sin embargo, también se pueden mezclar y dar paso a la hibridación de modelos (Hurtado Ruiz-Lara \& Argudo, 2020; Lamoneda, González-Víllora \& Fernández-Río, 2020; Quintero, Jiménez \& Area, 2018). Es importante resaltar que, los modelos pedagógicos se transforman en función de las solicitudes que la sociedad plasma a la educación física y estas a su vez están subordinadas al ambiente en el que va a germinar, sin olvidar que estas tendencias pueden variar con el paso del tiempo.

En cuanto al modelo que concierne, el AC, es de suma importancia para la educación física, entrando en las metodologías activas, según diversos autores (Hernández \& Cañabate, 2020; León-Díaz, ArijaMediavilla, Fernando-Martínez \& Santos-Pastor, 2020; Posso-Pacheco, et al., 2020). Entre las ventajas están las siguientes: fomenta la interacción, potencia la autonomía e independencia, promueve el desarrollo de destrezas complejas de pensamiento crítico, ayuda en el desarrollo socioafectivo, contribuye a reducir la violencia en la escuela, entre otras (Pérez, 2010)

Es necesario recalcar que existen diversas investigaciones que resaltan la importancia de implementar el AC en educación física (Velázquez, 2018). Este sirve para promover el aprendizaje motor (André, 2012; Bähr, 2010); desarrollar habilidades socio afectivas (FernándezRio, \& Méndez-Giménez, 2015); mejora el compromiso de los jóvenes con la educación física y su participación (Goodyear, Casey, \& Kirk, 2014); promueve un ambiente de tolerancia y respeto a la diversidad (Enríquez, Mera, \& Castro, 2016); favorece la inclusión del alumnado con capacidades distintas (Heredia \& Duran, 2013; Munafo, 2016); motiva a los estudiantes hacia la práctica motriz (Barba, 2015); permite incorporar el desarrollo de un segundo idioma a través de la educación física (Coral \& Lleixà, 2015); aumenta la participación de un evento deportivo no competitivo (León, Gamonales, Gómez-Carmona, Leal-Bello \& MuñozJiménez, 2019); puede tratarse cualquier bloque de contenidos de la educación física (Navarro-Paton, Rego \& García, 2017), entre otras cosas, y que a su vez ayudan al desarrollo de las competencias que necesitan los y las estudiantes. Debido a las ventajas mencionadas, se deduce que el AC es un método de trabajo extremadamente útil y provechoso si se utiliza en educación física (Herrero-González, López-Pastor \& Manrique-Arribas, 2020; Velázquez-Callado, 2015).

Habría que decir también que, de acuerdo con el pensamiento actual de investigación en la educación física, hay evidencia que demuestra que trabajar en grupos pequeños a través del $\mathrm{AC}$, si se les instruye correctamente, es mejor que las formas tradicionales de enseñanza, influyendo positivamente en el desarrollo de los alumnos (Groeben \& Wibowo, 2016; Lee, 2014; Lorente \& Martos, 2018). El concepto instruir correctamente significa que para aplicar el AC en la escuela de forma correcta se necesita una orientación competente 
y una supervisión por parte del docente. La necesidad de «hacerlo bien» (por así decirlo) puede requerir la adquisición de ciertas competencias por parte de los docentes y, con toda probabilidad, un cambio en el estilo de enseñanza en ciertos casos. Eso es si quieren ensenar cooperativamente.

A su vez, recientemente se está hablando del «Ciclo del Aprendizaje Cooperativo, el cual se recomienda mezclar con otros modelos pedagógicos» (FernándezRío, 2016, p. 265). Las tres fases de este ciclo son: 1creación y cohesión de grupo, 2- el AC como contenido para enseñar y aprender y 3- el AC como recurso para enseñar y aprender. Este ciclo no es una estructura piramidal vertical en la que se pasa de una fase a otra sin posibilidad de regreso. Al contrario, los profesores pueden y deben volver de una fase a otra según las necesidades de la clase para fortalecer determinados objetivos. En pocas palabras, este ciclo es un marco estable que les permite a los profesores y a sus estudiantes comprender, aprender y usar de una manera progresiva el $\mathrm{AC}$ en sus clases.

\section{Responsabilidad individual y $A C$}

En el AC existen cinco elementos esenciales para que sea viable: «interdependencia positiva claramente percibida, responsabilidad individual, interacción de promoción, habilidades sociales y procesamiento grupal» (Johnson \& Johnson, 2014, p.845). Aunque estos cinco elementos son importantes, este artículo de investigación se centrará en la responsabilidad individual. Esto debido a que cuando se trabaja en equipos, algunos estudiantes suelen apoyarse, por así decirlo, en el trabajo de otro, siendo este uno de los mayores retos en el AC. Es decir, no quieren trabajar, esperando que las actividades o tareas las realice otro.

Este modelo pedagógico, si se aborda correctamente, tiene la fortaleza de provocar en los estudiantes un aumento de la responsabilidad individual (Solano, 2015; Tamargo \& Rodríguez, 2015). Nadie se aprovecha del trabajo de otros. Todos aportan su granito de arena, por decirlo así. El equipo asume la responsabilidad de alcanzar sus objetivos, y cada miembro se hace responsable de cumplir con la parte del trabajo que le corresponde. (Marcillo, Núñez, Acuña, \& Beltrán, 2020; Martínez \& Sánchez, 2020). En palabras de Lago, Pujolàs, Riera y Vilarrasa, (2015, p.77): «se incrementa considerablemente la participación y la motivación del alumnado para la realización de las diferentes actividades propuestas para el grupo».

Como se puede ver, se han encontrado estudios en diversos países y regiones sobre el AC en educacion física. Sin embargo, todavía no se han hecho este tipo de investigaciones en la República Dominicana, por lo que surge una gran preocupación, ya que de no utilizarse esta metodología en nuestro país los docentes y estudiantes se estarían perdiendo de los excelentes resultados que este promete.

\section{Método}

\section{Tipo de estudio y diseño}

Este fue un estudio cuasiexperimental, solo con post test, puesto que no se asignaron al azar los sujetos a los grupos, sino que se utilizaron los grados y secciones existentes. Además de confirmar las características psicométricas de las escalas utilizadas para medir el AC, se hicieron comparaciones de los puntajes obtenidos en las escalas entre el grupo experimental, el cual recibió un entrenamiento en AC, y el grupo control, el cual no recibió ese entrenamiento. La hipótesis planteada fue que los participantes del grupo experimental obtendrían puntuaciones más altas que los del grupo control en la responsabilidad individual.

\section{Muestra}

Los participantes del grupo experimental fueron 104 estudiantes del Politécnico Mercedes Peña, quienes recibieron el entrenamiento cooperativo y luego llenaron el Cuestionario de Aprendizaje Cooperativo (CAC). Los participantes del grupo control fueron 154 estudiantes del Politécnico Milagros Celeste Arias, quienes no recibieron el entrenamiento cooperativo, pero sí llenaron el CAC.

Los dos grupos fueron considerados como homogéneos por provenir de politécnicos en los grados primero, segundo y tercero de secundaria. Ambos centros públicos pertenecen al mismo distrito escolar y están situados en barrios de igual nivel socioeconómico, medio bajo.

\section{Análisis}

Para poder validar las escalas del CAC se realizó un Análisis Factorial Confirmatorio (AFC) con el software Amos 22 para comprobar que se mantenían los 5 factores propuestos para el CAC por Fernandez-Rio, Cecchini, Mendez-Gimenez, Mendez-Alonso, \& Prieto, 2017. Para cada una de las 5 subescalas se calcularon los índices de confiabilidad compuesta (CC) para medidas congénicas con el calculador de Colwell (2016).

Las medias para cada una de las 5 subescalas fueron 
comparadas entre los dos grupos, experimental y control, a través de pruebas $t$ para muestras independientes usando el SPSS 22.

\section{Descripción de las intervenciones con $A C$}

Siguiendo las recomendaciones de Martínez \& Sánchez en el 2020, al inicio se planificó una charla de los beneficios del AC. Siempre tratando de utilizar las «técnicas de aprendizaje cooperativo» (Velázquez, 2013). En primer lugar, se les asignó una «tarea en equipo», luego se dividió a la clase en grupos de cinco estudiantes y después se le pidió a cada grupo que preparara una presentación de los beneficios del AC. Para asegurar la responsabilidad individual se asignó a cada miembro del grupo un rol específico y se le evaluó por ello. Los equipos heterogéneos formados, se mantuvieron así durante toda la unidad didáctica.

En segundo lugar, se utilizó la técnica de comprobación en parejas o «Pair Check». Los estudiantes se distribuyen en grupos de cuatro personas. Dentro de cada grupo se forman dos parejas. Al finalizar una parte de la explicación sobre los tipos de aperturas en ajedrez, el docente presenta una lista de ejercicios o problemas de ajedrez. Uno de los miembros de cada pareja lee el primer ejercicio y explica, paso por paso, cómo resolverlo, lo hace y da la solución. Su compañero desempeña el rol de instructor y verifica que el proceso y la solución sean correctos. En caso de dudas pueden pedir ayuda a la otra pareja que completa su grupo y, en caso de que también tenga problemas, los cuatro estudiantes levantan sus manos para solicitar la ayuda del docente. Luego, los dos estudiantes que componen cada pareja intercambian sus papeles para el siguiente ejercicio. Cuando la pareja resuelve dos problemas, verifican sus respuestas con la otra pareja que compone su grupo. Si hay discrepancias, se analizan las respuestas hasta alcanzar un consenso; si están de acuerdo, se agradecen mutuamente la ayuda prestada y continúan trabajando en parejas.

En tercer lugar, se empleó la técnica de piensa forma pareja - comparte o «Think - Pair - Share». Esta técnica constó de tres fases: primero, el docente plantea una o varias preguntas a la clase sobre las aperturas abiertas y semiabiertas. Cada estudiante piensa individualmente la respuesta a dichas preguntas; segundo, cada estudiante forma pareja con otro compañero y comparten sus respectivas respuestas tratando de alcanzar un consenso; y tercero, cada pareja comparte su respuesta consensuada con la clase, buscando alcanzar un consenso general.
En cuarto lugar, se usó la técnica cabezas numeradas juntas o «Numbered Heads Together». Se divide a la clase en grupos. Dentro de cada grupo, cada uno de sus miembros tiene un número diferente. El docente pide a los grupos que resuelvan un problema sobre las aperturas cerradas en ajedrez y da un tiempo determinado para hacerlo. Al finalizar el tiempo convenido, dice un número y la persona de cada uno de esos grupos con ese número levanta su mano. El docente pregunta a una de ellas por la solución. Como ninguno de los grupos sabe qué número dirá el profesor, todos se esfuerzan por compartir la información, buscando que, sea quien sea, la persona que los represente sepa explicar la respuesta correcta al problema planteado. Si la respuesta es correcta, su equipo recibe un punto; en caso contrario, el docente pregunta a un estudiante de otro grupo.

En quinto lugar, otra técnica utilizada fue el repaso cooperativo en grupos. Se divide la clase en grupos. El día anterior al examen sobre la Historia del voleibol (internacional, nacional y local), reglas elementales, posturas fundamentales, desplazamientos, saque, recepción, voleo, ataque, bloqueo y defensa, los distintos grupos se turnan para hacer preguntas de repaso a los otros equipos, estableciéndose con anterioridad un sistema de puntuación. El equipo que pregunta obtiene un punto por cada pregunta que formula. El grupo que debe contestar obtiene un punto si lo hace correctamente. Un segundo grupo puede ganar un punto si agrega información importante a la respuesta dada por el grupo anterior. El grupo que pregunta lo hace según un orden establecido, hasta que el equipo interrogado formula la respuesta correcta. Gana el equipo con mayor puntuación.

Para terminar, también de aplicaron las siguientes técnicas: mesa redonda o «Roundtable», discusión grupal espontánea, levántate y comparte o «stand and share», torneo de Juegos por equipos o «Teams-GamesTournament», finalmente pero no menos importante, el Rompecabezas, también llamado Puzle o «Jigsaw».

Los contenidos que se trabajaron fueron voleibol y ajedrez. Se formaron grupos heterogéneos en todas las secciones. Las clases tuvieron una duración de tres meses, con dos sesiones por semana, para un total en promedio de 24 sesiones de clase. Se trabajó en los grados de primero, segundo y tercero de secundaria. La edad en estos grados es de 12, 13 y 14 años, aproximadamente.

En cuanto a la evaluación se refiere, se utilizó la autoevaluación, heteroevaluación y coevaluación. Así, por ejemplo, en la autoevaluación, se animó a los estu- 
diantes a que se plantearan las siguientes preguntas: ¿He conseguido aprender o lograr realizar las propuestas gracias a mis compañeros y he ayudado para que ellos también puedan? ¿He apoyado, ayudado y animado a mis compañeros a mejorar? ¿He realizado perfectamente mi trabajo y he procurado que mi parte del trabajo haya contribuido directamente a los objetivos del grupo? ¿He desarrollado mis habilidades sociales (habilidades interpersonales, competencia afectiva, motivación, compromiso, empatía, paciencia) para el buen ambiente del grupo? ¿He participado activamente en el proceso de autoevaluación grupal, analizando lo que ha ocurrido para continuar mejorando? ¿He permitido o favorecido que todos tengamos las oportunidades necesarias para aprender y tener éxito? ¿Me he preocupado de que todos hayamos participado en función de nuestras posibilidades?

En ese mismo orden de ideas, en la coevaluación se les presentaba una escala de clasificación para evaluar el gesto técnico correcto del toque de dedos y de antebrazo en voleibol. El valor 0, era otorgado si lo hacía muy mal, el valor 1 regular, y finalmente el 2 muy bien. En la figura 1 se encuentra el formulario para dicha prueba.

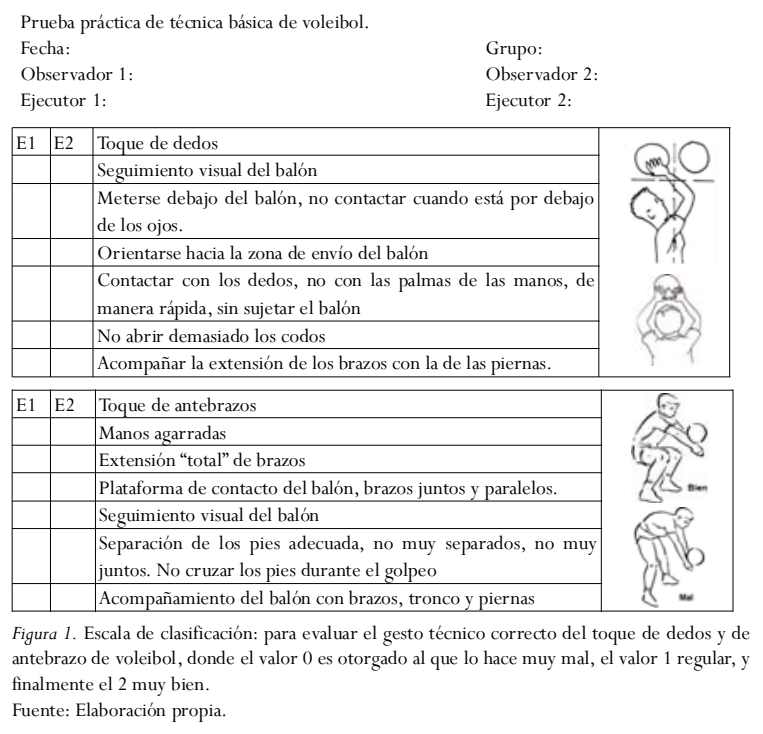

\section{Instrumentos}

En esta investigación se ha utilizado como instrumento el CAC, que ya ha sido previamente validado y utilizado por Fernandez-Rio et al. (2017).

El CAC tiene un total de 20 ítems, a razón de 4 ítems en cada una de las 5 dimensiones planteadas. El formato de respuesta fue la escala Lickert de 5 puntos (desde $1=$ totalmente en desacuerdo, a $5=$ totalmente de acuerdo) por su adecuación a la edad de los encuestados y su idoneidad para que se considere válido en estadística (Soriano, 2014). Tiene una raíz común a todos los ítems al comienzo del cuestionario: «En clase...». Con el objetivo de asegurar tanto la validez de contenido como la aplicabilidad del instrumento, el cuestionario fue sometido a un doble proceso de depuración, juicio de expertos y estudio piloto. (FernandezRio, et al. 2017).

En la validación mencionada el cuestionario mostró estar compuesto por 5 factores o componentes: Habilidades Sociales (HS), Procesamiento Grupal (PG), Interdependencia Positiva (IPos), Interacción Promotora (IPro), y Responsabilidad Individual (RI).

\section{Resultados}

\section{Características psicométricas del CAC}

En la Tabla 1 comparamos los índices de ajuste del modelo obtenidos en el AFC en el presente estudio con los obtenidos por Fernandez-Rio et al. (2017).

Tabla 1.

\begin{tabular}{lccc}
\hline \multicolumn{4}{l}{ Comparación de los indices de ajuste del modelo teórico en el AFC. } \\
Fernandez-Rio et al., & & \\
Índices de ajuste & 2017 & Presente estudio & Criterio \\
\hline CMIN & 2574.51 & 289.111 & \\
GL & 160 & 160 & \\
CMIN/GL & 16.1 & 1.81 & $<3$ \\
CFI & 0.953 & 0.91 & $>.90$ \\
RMSEA & 0.037 & 0.046 & $<.08$ \\
\hline Fuente: Elaboración propia en base a los datos del presente estudio y los reportados por
\end{tabular}

Fuente: Elaboración propia en base a los datos del presente estudio y los reportados por Fernandez-Rio et al., 2017

Tabla 2 .

\begin{tabular}{lllll} 
Descriptivas y carga factorial de cada item y confiabilidad de cada factor y escala total CAC. \\
\hline Factores & Media & DE & Cargas & CC
\end{tabular} \begin{tabular}{lrrrr} 
Factores & Media & DE $\begin{array}{c}\text { Cargas } \\
\text { factoriales }\end{array}$ \\
\hline Habilidades Sociales (HS) & 15.21 & 3.241 & 0.90
\end{tabular}

\begin{tabular}{lrrr}
\hline Habilidades Sociales (HS) & 15.21 & 3.241 & \\
1.Trabajamos el díálogo, la capacidad de escucha y/o el debate & 3.89 & 1.033 & 0.507
\end{tabular} 6.Exponemos y defendemos ideas, conocimientos y puntos de $\begin{array}{llll}3.83 & 1.118 & 0.678\end{array}$ vista ante los compañeros

11.Escuchamos las opiniones y los puntos de vista de los $\quad \begin{array}{llll}4.01 & 1.095 & 0.591\end{array}$ compañeros

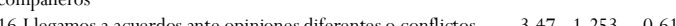
\begin{tabular}{lrrrr} 
16.Llegamos a acuerdos ante opiniones diferentes o conflictos & 3.47 & 1.253 & 0.612 & \\
\hline Procesamiento Grupal (PG) & 15.33 & 3.197 & & 0.90
\end{tabular}

2.Hacemos puestas en común para que todo el mundo conozca lo $\begin{array}{rrrr}3.97 & 1.071 & 0.564\end{array}$ que se está haciendo

7.Tomamos decisiones de forma consensuada entre los $\quad 3.68 \quad 1.194 \quad 0.531$ compañeros del grupo

12.Debatimos las ideas entre los miembros del grupo $\quad \begin{array}{llll}3.91 & 1.152 & 0.589\end{array}$ 17. Reflexionamos de manera individual y de manera conjunta $\begin{array}{llll}3.77 & 1.065 & 0.680\end{array}$ \begin{tabular}{lllll} 
dentro del grupo & & & & \\
\hline Interdependencia Positiva (IPOS) & 15.45 & 3.193 & 0.89
\end{tabular} \begin{tabular}{lcccc}
\hline Interdependencia Positiva (IPOS) & 15.45 & 3.193 & & 0.89 \\
3.Es importante la ayuda de mis compañeros para completar las & 3.69 & 1.322 & 0.608
\end{tabular} tareas

8.No podemos terminar una actividad sin las aportaciones de los $\quad \begin{array}{llll}3.43 & 1.259 & 0.531\end{array}$ compañeros

13.Es importante compartir materiales, información... para hacer $4.21 \quad 0.99 \quad 0.608$ 13. Es in

18. Cuanto mejor hace su tarea cada miembro del grupo, mejor $\quad 4.12 \quad 1.111 \quad 0.561$ resultados obtiene el grupo

Interacción Promotora (IPRO) $\quad 15.343 .119 \quad 0.87$

$\begin{array}{lllll}\text { 4.Los compañeros de grupo se relacionan e interactúan durante } & 3.80 & 1.078 & 0.420\end{array}$ las tareas

9.La interacción entre compañeros de grupo es necesaria para $\quad \begin{array}{llll}3.66 & 1.291 & 0.587\end{array}$ hacer la tarea

$\begin{array}{lllll}\text { 14.Nos relacionamos unos con otros para hacer las actividades } & 3.95 & 1.101 & 0.554\end{array}$ \begin{tabular}{lrrrrr} 
14. Nos relacionamos unos con otros para hacer las actividades & 3.95 & 1.101 & 0.554 & \\
19.Trabajamos de manera directa unos con otros & 3.93 & 1.039 & 0.635 & \\
\hline Responsabilidad Individual (RI) & 16.55 & 2.967 & & 0.90
\end{tabular} $\begin{array}{lllll}5 . C a d a & \text { miembro del grupo debe participar en las tareas del grupo } & 4.01 & 1.142 & 0.568\end{array}$ 10. Cada componente del grupo debe esforzarse en las actividades $4.21 \quad 0.983 \quad 0.621$ del grupo

15.Cada miembro del grupo debe tratar de participar, aunque no $\begin{array}{llll}4.11 & 1.028 & 0.613\end{array}$ le guste la tarea

20.Cada miembro del grupo debe hacer su parte del trabajo del $\quad 4.22 \quad 1.000 \quad 0.566$ grupo para completar la tarea

Escala Total CAC 77.8713 .01 $0.90 *$

*Nota: Alfa de Cronbach.

Fuente: Elaboración propia. 
En esta tabla vemos que la discrepancia mínima (CMIN) fue menor en el presente estudio. La misma, cuando se divide entre los grados de libertad (GL) nos ofrece un Chi-cuadrado relativo (CMIN/GL) cuyo criterio de buen ajuste es que debe ser menor de 3 . Nuestro estudio cubrió cómodamente el criterio, lo cual no ocurrió en el estudio de Fernández-Rio. Por otra parte, el Índice de Ajuste Comparativo (CFI) cumplió con el criterio de ser superior a .90 en ambos estudios. También, en el Índice Raíz del Error de Aproximación al Cuadrado (RMSEA), en ambos estudios se cumplió con el criterio. Este resultado nos indica que la validez de constructo de la escala CAC fue buena.

En la Tabla 2 se muestran las estadísticas descriptivas y la carga factorial de cada ítem, así como la confiabilidad compuesta (CC) de cada factor. La confiabilidad de la escala total fue un índice Alfa de Cronbach, calculado con el SPSS.

La validez convergente de la escala se comprueba porque todas las cargas factoriales fueron mayores de .50 , lejos del límite inferior admitido de .30. Además, todos los índices de confiabilidad fueron mayores del límite aceptable de .70, de hecho, desde .87 hasta .90 .

La correlación entre los factores se presenta en la Tabla 3. En esta tabla podemos constatar que la validez discriminante de los factores fue buena, porque ninguna de ellas fue mayor de .85 , todas fueron positivas y moderadas. En otras palabras, los factores o componentes no estaban midiendo el mismo constructo.

\begin{tabular}{|c|c|c|c|c|}
\hline \multicolumn{5}{|c|}{ Correlaciones entre los factores de la escala $C A C$. } \\
\hline & Habilidades & Procesamiento & Interdependencia & Interacción \\
\hline & Sociales & Grupal & Positiva & Promotora \\
\hline Procesamiento Grupal & 0.67 & & & \\
\hline Interdependencia Positiva & 0.57 & 0.59 & & \\
\hline Interacción Promotora & 0.56 & 0.62 & 0.61 & \\
\hline Responsabilidad Individual & 0.61 & 0.62 & 0.58 & 0.61 \\
\hline
\end{tabular}

Diferencias entre el grupo experimental y el grupo control

La Figura 2 muestra las diferencias en las puntuaciones en el CAC entre el grupo experimental (Politécnico Mercedes Peña) y el grupo control (Politécnico Milagros Celeste Arias). En esta figura podemos apreciar que las medias de puntuaciones en el CAC siempre fueron más altas en el grupo experimental. Sin embargo, la única diferencia estadísticamente significativa fue encontrada en el factor Responsabilidad Individual, $t$ (256) $=2.082, p=.038$, con un tamaño del efecto pequeño $(d=.26)$ y una potencia de la prueba baja $(1-\hat{a}=.53)$. Ninguna de las diferencias entre los demás factores mostró significación estadística.

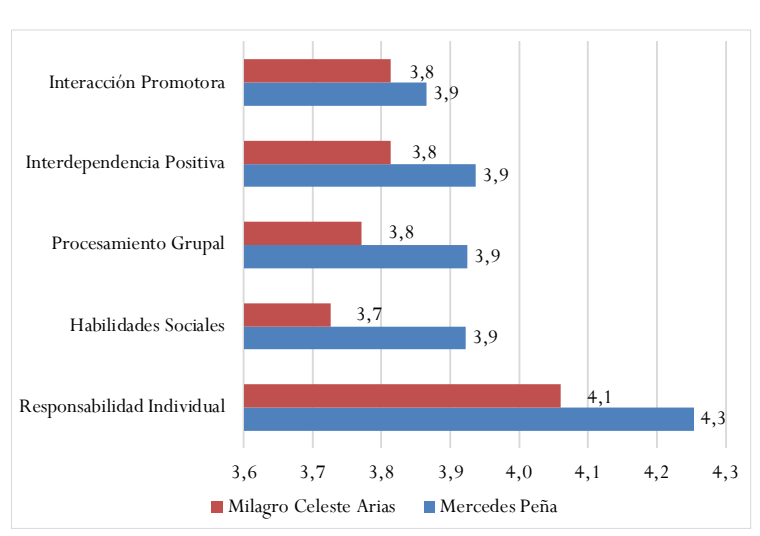

Figura 2. Medias en la escala CAC en los grupos experimental y control. Fuente: Elaboración propia.

\section{Discusión y conclusión}

De acuerdo con los resultados obtenidos, el entrenamiento en AC consiguió una incidencia notable en las competencias necesarias para que dicho modelo pedagógico gozara de éxito en las clases de educación física, aunque el factor Responsabilidad Individual fue el que tuvo un mayor aumento (Fernández-Rio, \& MéndezGiménez, 2015).

En ese mismo orden, al culminar las clases con este modelo pedagógico, hemos constatado que nuestros estudiantes necesitan cumplir con algunos requisitos, tales como: tiene que existir un contacto directo entre los participantes, sólo tienen éxito si los compañeros de su grupo también lo tienen; cada integrante del grupo debe tener una función que desempeñar; buena comunicación entre un grupo y demostrar una escucha activa, respetando el turno de los demás (Bermejo, et al., 2020). Se ha visto, como los estudiantes asumen un papel activo, facilitándole la ayuda necesaria al resto de sus compañeros para lograr una meta en común y no se limitaban solamente a aprender un contenido. (Arís, 2015; García, Sánchez \& Ferriz, 2020; Martínez-Benito, 2017; Navarro, Pellicer \& Collado 2020; Pérez \& Hortigüela,2019). Claro está, este cambio de una cultura competitiva a una cooperativa es gradual, por lo que todavía en los siguientes años se podrán ver sus frutos.

Es importante mencionar que, en un inicio, muchos estudiantes eran sumamente competitivos, mayormente terminaban en discordia con sus compañeros cuando trabajaban en equipo, ya que no asumían la responsabilidad de sus errores. Eso cambió en el grupo experimental después de aplicar el AC. Lo cual indica, que las clases cooperativas sí mejoraron notablemente la responsabilidad individual, cumpliéndose así la hipótesis planteada. Motivo por el cual, este modelo pedagógico 
es recomendable para usarlo en educación física.

Por otro lado, se recomienda que se hagan intervenciones y entrenamientos cooperativos por más tiempo. Se notó que los estudiantes no estaban muy familiarizados con dicho modelo pedagógico. Es necesario que nuestros estudiantes aprendan a cooperar, pues dentro de poco van a pasar a la adultez. Es bueno que desde ahora se familiaricen con la cultura cooperativa.

Para una futura investigación, exhortamos a la hibridación de otros modelos pedagógicos con el AC (Hurtado, Ruiz-Lara \& Argudo, 2020; Lamoneda Prieto, González-Víllora \& Fernández-Río, 2020; Quintero, Jiménez \& Area, 2018). Con esto queremos decir, implementar varios modelos pedagógicos a la vez y no uno solo, porque al mezclar dos o más, se fortalecen sus beneficios educativos, se minimizan algunas de sus restricciones y, se podría implementar una educación más adaptada e individualizada de acuerdo con el contexto en que se lleve a cabo.

Para terminar, se ha comprobado que gracias al AC nadie se aprovecha del trabajo de otros. El equipo asume la responsabilidad de alcanzar sus objetivos, y cada miembro se hace responsable de cumplir con la parte del trabajo que le corresponde, lo que en general mejora el clima del aula (Martínez \& Sánchez, 2020). Es imperativo que este modelo pedagógico se siga implementando en la educación física de la República Dominicana para que los estudiantes sigan desarrollando las competencias necesarias para un buen aprendizaje a lo largo de su recorrido por la vida, no solamente en el centro educativo.

\section{Agradecimientos}

Agradecemos infinitamente al Instituto Superior de Formación Docente Salomé Ureña, (ISFODOSU), por financiar esta investigación, y así contribuir a la mejora e innovación de las prácticas educativas en la República Dominicana. También a los politécnicos Mercedes Peña y Milagros Celeste Arias, por abrirnos sus puertas para esta investigación. Asimismo, las gracias a la revista Retos: nuevas tendencias en educación física, deporte y recreación, por brindarnos la oportunidad de publicar nuestro artículo con ustedes. Finalmente, a todos los que contribuyeron, ya sea directa o indirectamente, para que este proyecto tuviera éxito.

\section{Referencias}

Anderman, E., \& Dawson, H. (2011). Learning with
Motivation. En R. E. Mayer, y P. A. Alexander, Handbook of Research on Learning and Instruction. 219-242. New York.

André, A. (2012). Influence de l'Apprentissage Coopératif sur le savoir s'échauffer et la motivation autodéterminée vis à vis de l'échauffement, eJRIEPS, 27. https://doi.org/10.4000/ejrieps. 3048

Arís, N. (2015). Aprendizaje cooperativo y logro competencial. Opción, 31(2), 81-88. Recuperado de: https: / / www.redalyc.org / articulo.oa?id=310/ 31045568006

Bähr, I. Experiencia práctica y resultados empíricos sobre el aprendizaje cooperativo en gimnasia. In: Velázquez, Carlos (Coord.). Aprendizaje cooperativo en Educación Física. Fundamentos y aplicaciones prácticas. Barcelona: INDE, 2010, 149-163.

Barba, J. (2015). Diferencias entre el aprendizaje cooperativo y la asignación de tareas en la Escuela Rural. Comparación de dos estudios de caso en una unidad didáctica de acrosport en segundo ciclo de primaria. Retos, 18, 14-18. https://doi.org/ 10.47197/retos.v0i18.34644

Bermejo, J., Pulido, D., Galmés, A., Serra, P., Vidal, J., \& Ponseti, F. (2020). Educación física y universidad: Evaluación de una experiencia docente a través del aprendizaje cooperativo. Retos, 39, 90-97. https:/ / doi.org/10.47197/retos.v0i39.77834

Colwell, R. (2016). The composite reliability calculator. Technical Report, DOI: 10.13140/ RG.2.1.4298.088.

Coral, J., \& Lleixà, T. (2015). Las tareas en el aprendizaje integrado de educación física y lengua extranjera (AICLE). Determinación de las características de las tareas mediante el análisis del diario de clase. Retos, 24, 79-84. https://doi.org/10.47197/ retos.v0i24.34532

Enríquez, L. C., Mera, O. E., \& Castro, I. E. (2016). El aprendizaje cooperativo como alternativa para la inclusión en la Educación Física. RECUS. 1(3), 1-5. Recuperado de https://revistas.utm.edu.ec/ index.php/Recus/article/download/52/38/

Feria-Madueño, A.; Grimaldi-Puyana, M., SánchezOliver, A. J., \& Alcaraz-Rodríguez, V. (2017). Aprendizaje cooperativo y su percepción en futuros docentes de educación física. Trances, 9 (1): 189-198. Recuperado de

h t t p : / / w w w.trances.es / papers / TCS_09_supl01_01.pdf

Fernández-Río, J., Calderón, A., Hortigüela, D. , PérezPueyo, A., \& Aznar, M. (2016). Modelos pedagógi- 
cos en educación física: consideraciones teórico-prácticas para docentes. Revista Española de Educación Física y Deportes, 413, 55-75. Recuperado de: https: / / www.reefd.es/index.php/reefd/article/view/425

Fernández-Rio, J., \& Méndez-Giménez, A. (2015). El Aprendizaje Cooperativo: Modelo Pedagógico para Educación Física. Retos, 29, 201-206. https: / / doi.org/ 10.47197/retos.v0i29.38721

Fernandez-Rio, J., Cecchini, J. A., Mendez-Gimenez, A., Mendez-Alonso, D., \& Prieto, J. A. (2017). Diseño y validación de un cuestionario de medición del aprendizaje cooperativo en contextos educativos. Anales De Psicología, 33(3), 680-688. https:// doi.org/10.6018/analesps.33.3.251321

Fernández-Río, J. (2016). El Ciclo del Aprendizaje Cooperativo: una guía para implementar de manera efectiva el aprendizaje cooperativo en educación física. Retos, 32, 264-269. https: / / doi.org/10.47197/ retos.v0i32.51298

García, S., Sánchez, P., \& Ferriz,A. (2020). Metodologías cooperativas versus competitivas: efectos sobre la motivación en alumnado de EF. Retos, 39, 65-70. https: / / doi.org/10.47197/retos.v0i39.78279

Goodyear, V. A., Casey, A., \& Kirk, D. (2014). Hiding behind the camera: social learning within the cooperative learning model to engage girls in physical education. Sport, Education and Society, 19(6), 712-734. https://doi.org/10.1080/ 13573322.2012.707124

Groeben, B., \& Wibowo, J. (2016). Empirical Contributions to the practice of Cooperative Learning in Physical Education. En U. A. (UAP), Actes 10 Congrés Internacional d'Activitats Físiques Cooperatives, 822-833. Barcelona: Institut de Ciències de l'Educació de la Universitat Autònoma de Barcelona. Recuperado de https: / / www.uab.cat/ doc/Actes_10_Congres_Internacion al_Activitats_Fisiques_Cooperatives

Heredia, J., \& Duran, D. (2013). Aprendizaje cooperativo en educación física para la inclusión de alumnado con rasgos autistas. Revista nacional e internacional de educación inclusiva, 6(3), 25-40. Recuperado de http:/ /revistaeducacioninclusiva.es/index.php/REI/ article/view/143/137

Hernández, A., \& Cañabate, D. (2020). Role-playing y diálogo como protagonistas en la evaluación entre iguales: una experiencia de aprendizaje cooperativo. Sportis. Scientific Journal of School Sport, Physical Education and Psychomotricity, 6(2), 182-203. https: / / doi.org/10.17979/sportis. 2020.6.2.6041
Herrero-González, D., López-Pastor, V., \& ManriqueArribas, J. (2020). La Evaluación Formativa y Compartida en contextos de Aprendizaje Cooperativo en Educación Física en Primaria. Cultura, Ciencia y Deporte, 15(44), 213-222. Recuperado de: https: / / dialnet.unirioja.es/descarga/articulo/7427876.pdf

Hurtado, C., Ruiz-Lara, E., \& Argudo, F. (2020). Efectos del Aprendizaje Cooperativo y Modelo de Responsabilidad Personal y Social sobre el aprendizaje competencial y el clima motivacional percibido del alumnado de Educación Física en Primaria. Trances, 12(5), 612- 637. Recuperado de: https:// d i a l n e t. un i r i ja.es / s e r v let/ articulo?codigo $=7680267$

Johnson, D. W., Johnson, R. T., \& Smith, K. A. (2014). Cooperative learning: Improving university instruction by basing practice on validated theory. Journal on Excellence in College Teaching, 25(3\&4), 85118. Recuperado de https:// www.researchgate.net/publication/284471328

Johnson, D. W., \& Johnson, R. T. (2014). Cooperative Learning in 21st Century. [Aprendizaje cooperativo en el siglo XXI]. Anales De Psicología / Annals of Psychology, 30(3), 841-851.https://doi.org/10.6018/ analesps.30.3.201241

Lago, J. R., Pujolàs, P., Riera, G., \& Vilarrasa, A. (2015). El aprendizaje cooperativo y cómo introducirlo en los centros escolares. Revista Latinoamericana, 9(2), 73-90. Recuperado de https:/ / dialnet.unirioja.es/ servlet/articulo?codigo $=5504531$

Lamoneda, J., González-Víllora, S., \& Fernández-Río, J. (2020). Hibridando el Aprendizaje Cooperativo, la Educación Aventura y la Gamificación a través de la carrera de orientación. Retos, 38, 754-760. https: / /doi.org/10.47197/retos.v38i38.77276

Lee,T. (2014). Effects of a Cooperative Learning Strategy on the Effectiveness of Physical Fitness Teaching and Constraining Factors. Mathematical Problems in Engineering, Article ID 519291, 1-6. https:/ / doi.org/ $10.1155 / 2014 / 519291$

León-Díaz, O., Arija-Mediavilla, A., Martínez-Muñoz, L., \& Santos-Pastor, M. (2020). Las metodologías activas en Educación Física. Una aproximación al estado actual desde la percepción de los docentes en la Comunidad de Madrid. Retos, 38, 587-594. https: / /doi.org/10.47197/retos.v38i38.77671

León, K., Gamonales, J., Gómez-Carmona, C. D., LealBello, G., \& Muñoz-Jiménez, J. (2019). Análisis de los factores que influyen en la cooperación deportiva en las actividades gimnásticas grupales no com- 
petitivas. Retos, 37, 532-539. https://doi.org/ 10.47197/retos.v37i37.71399

Lorente, E., \& Martos, D. (2018). Educación Física y pedagogía crítica: Propuestas para la transformación personal y social. Universidad de Lleida. 273-292.

Marcillo, J., Núñez, L., Acuña, M., \& Beltrán, M. (2020). El desarrollo de los enfoques curriculares de educación física a través del aprendizaje cooperativo. Revista EDUCARE - UPEL-IPB - Segunda Nueva Etapa 2.0, 24(2), 145-166. https://doi.org/10.46498/ reduipb.v24i2.1324

Martínez, R., \& Sánchez, G. (2020). El Aprendizaje Cooperativo en la clase de Educación Física: dificultades iniciales y propuestas para su desarrollo. Revista Educación, 44(1). https://doi.org/10.15517/ revedu.v44i1.35617

Martínez-Benito, R., (2017). Aprendiendo a trabajar en equipo en el aula: una propuesta basada en el aprendizaje cooperativo y en la evaluación formativa en educación física. XII Congreso Internacional Sobre La Enseñanza De La Educación Física Y El Deporte Escolar, 472-480. Recuperado de: https: / / www.researchgate.net/publication/321017154

Munafo, C. (2016). Cooperative Learning as Formative Approach in Physical Education for All. International Journal of Science Culture and Sport, 4(2), 195-205. DOI: 10.14486/INTJSCS513

Navarro, D., Pellicer, I., \& Collado, J. (2020). Modelos Pedagógicos en Educación Física / Daniel Navarro Ardoy, Juan Ángel Collado Martínez, Irene Pellicer Royo ( $1^{\mathrm{a}} \mathrm{ed}$.). Independently published. Aprendizaje cooperativo, ¿quieres llegar rápido o lejos? 95-119.

Navarro-Paton, R., Rego, B., \& García, M. (2017). Incidencia de los juegos cooperativos en el autoconcepto físico de escolares de educación primaria. Retos, 34, 14- 18 . https://doi.org/10.47197/ retos.v0i34.58803

Pérez, A., \& Hortigüela, D. (2019). ¿Y si toda la innovación no es positiva en Educación Física? Reflexiones y consideraciones prácticas. Retos, 37, 579-587. https://doi.org/10.47197/retos.v37i37.74176

Pérez, S. (2010). El aprendizaje cooperativo. Revista digital para profesionales de la enseñanza, 8, 1-6. Recuperado de https://www.feandalucia.ccoo.es / docuipdf.aspx?d=7194\&s=

Posso-Pacheco, R., Barba-Miranda, L., Rodríguez-Torres, A., Núñez-Sotomayor, L., Ávila-Quinga, C., \& Rendón-Morales, P. (2020). An Active Microcurricular Learning Model: A Guide to Classroom Planning for Physical Education. Revista
Electrónica Educare, 24(3), 1-18. https://doi.org/ 10.15359/ree.24-3.14

Pujolàs, P. (2012). Aulas inclusivas y aprendizaje cooperativo. Educatio Siglo XXI, 30(1), 89-112. Recuperado de: https: / / revistas.um.es/educatio/article/ view/149151

Quintero, L., Jiménez, F., \& Area, M. (2018). Más allá del libro de texto. La gamificación mediada con TIC como alternativa de innovación en Educación Física. Retos, 34, 343-348. https://doi.org/10.47197/ retos.v0i34.65514

Solano, L. (2015). Influencia en el aprendizaje de los alumnos de $1^{\circ}$ y $2^{\circ}$ de Educación Secundaria Obligatoria del Aprendizaje Cooperativo. Indivisa. Boletín de Estudios e Investigación, 15, 51-76. Disponible en: https: / / www.redalyc.org / articulo. oa?id=771 / 77137915006

Soriano, A. (2014). Diseño y validación de instrumentos de medición. Diá-logos 14, 19-40. Recuperado de https: / / www.lamjol.info/index.php/DIALOGOS/article/view/2202

Tamargo, P. M., \& Rodríguez, C. (2015). Implicaciones del aprendizaje cooperativo en educación secundaria obligatoria. Revista de Estudios e Investigación en Psicología y Educación, (01), 109-114. https: / / doi.org/ 10.17979/reipe.2015.0.01.547

Velázquez, C. (2013). Análisis de la implementación del aprendizaje cooperativo durante la escolarización obligatoria en el área de educación física.

Universidad de Valladolid. Facultad de Educación y Trabajo Social. DOI: 10.35376/10324/2823

Velázquez-Callado, C. (2015). Aprendizaje cooperativo en Educación Física: estado de la cuestión y propuesta de intervención. Retos, 28, 234-239. https:// doi.org/10.47197/retos.v0i28.35533

Velázquez-Callado, C. (2015). Enfoques y posibilidades del aprendizaje cooperativo. Tándem Didáctica de la Educación Física, 50, 25-31.

Recuperado de: https: / /www.researchgate.net/ publication/299597300

Velázquez, C. (2018). El aprendizaje cooperativo en educación física: Planteamientos teóricos y puesta en práctica. Acción motriz, 20, 7-16. Recuperado de http: / /www.accionmotriz.com/documentos/revistas/articulos/20_1.pdf 